\title{
Sistematización y Trabajo Social en Chile. El largo y sinuoso camino
}

\section{Patricia Lorena Castañeda-Meneses}

Doctora en Ciencias de la Educación. Trabajadora Social

Universidad de Valparaíso. Valparaíso, Chile

https:/ / orcid.org/0000-0002-4676-5872・patricia.castaneda@uv.cl

\author{
Ana María Salamé-Coulon \\ Doctora en Ciencias de la Educación. Trabajadora Social \\ Universidad de La Frontera. Temuco, Chile \\ https:/ / orcid.org/0000-0001-6650-7507• ana.salame@ufrontera.cl
}

Resumen

Las tardías oportunidades que representa la sistematización para el Trabajo Social chileno son resultado de un lento proceso de reconocimiento de su potencial como propuesta de construcción de conocimientos, originado en las adversas condiciones derivadas del contexto nacional y de la subvaloración académica que ha debido enfrentar a lo largo de su desarrollo teórico metodológico. En ese marco, el presente artículo tiene por objetivo exponer los principales antecedentes del desarrollo histórico de la sistematización en Chile a fin de comprender en perspectiva la actual posición de desventaja que ocupa en el país.

Palabras clave: Trabajo Social chileno; Sistematización; Desarrollo histórico de la Sistematización; Formación profesional; Desempeño profesional.

Recibido: 28/08/2020 |Aprobado: 21/11/2020 | Publicado: 01/01/2021

(1) (1)(2) Esta obra está bajo una Licencia Creative Commons Atribución-NoComercialCompartirIgual 4.0 Internacional.

¿Cómo citar este artículo? / How to quote this article?

Castañeda-Meneses, P. L., y Salamé-Coulon, A. M. (2021). Sistematización y Trabajo Social en Chile. El largo y sinuoso camino. Prospectiva. Revista de Trabajo Social e intervención social, (31), 115-129. doi: 10.25100/prts.v0i31.10563. 
Castañeda-Meneses y Salamé-Coulon

\title{
Systematization and Social Work in Chile. The long and winding road
}

\begin{abstract}
The late opportunities that systematization represents for Chilean social work are the result of a slow process of recognition of its potential as a knowledge construction proposal, originated in the adverse conditions derived from the national context and from the academic undervaluation that it has had to face throughout its theoretical and methodological development. In such a framework, this article aims to present the main antecedents of the historical development of systematization in Chile in order to understand, in perspective, the current disadvantageous position it occupies in the country.

Keywords: Chilean Social Work; Systematization; Systematization historical development; Academic training; Professional performance.

Sumario: 1. Introducción, 2. Reflexión teórico-conceptual, 2.1 Antecedentes históricos de la Sistematización en Chile. Desde un camino abierto a un sendero clandestino, 2.2 Situación actual de la Sistematización en Chile. Avanzar desde los senderos del miedo y la indiferencia hasta retomar la propia travesía, 3. Conclusiones, 4 . Referencias bibliográficas.
\end{abstract}


Castañeda-Meneses y Salamé-Coulon

\section{Introducción}

“El largo y sinuoso camino que conduce a tu puerta nunca desaparecerá. Ya he visto este
camino antes. Siempre me trae aquí. Me conduce a tu puerta" Lennon \& McCartney (1970)

\section{El largo y sinuoso camino que conduce a tu puerta}

Las tardías oportunidades que representa la Sistematización para el Trabajo Social chileno son resultado de un lento proceso de reconocimiento de su potencial como estrategia de construcción de conocimientos, originado en las adversas condiciones derivadas del contexto nacional y de la subvaloración académica que ha debido enfrentar a lo largo de su desarrollo teórico metodológico. En consecuencia, hablar de Sistematización en el Trabajo Social chileno es transitar por caminos inestables, debido a que no hay acuerdo pleno respecto de sus aportes especializados, situación expresada en frecuentes desconfianzas respecto de su verdadero potencial para contribuir a la construcción de conocimientos y en permanentes cuestionamientos en torno al rigor metodológico de sus pautas de trabajo o respecto de la validez y confiabilidad de sus resultados. En ese marco, el presente artículo tiene por objetivo exponer los principales antecedentes del desarrollo histórico de la Sistematización en Chile, con el fin de comprender en perspectiva la actual posición de desventaja que ocupa en los contextos académicos y profesionales del país. Para la realización del artículo se realizó revisión y análisis documental de textos académicos, materiales de trabajo y publicaciones especializadas que refieren directamente a las particularidades del desarrollo, avances y retrocesos que ha experimentado la Sistematización, en su búsqueda de legitimidad y reconocimiento. La estructura del artículo a partir de esta introducción se inicia con un primer apartado que presenta los antecedentes históricos de la Sistematización en Chile, destacando especialmente la época dictatorial y el retorno a la democracia como contextos que influyen directamente en la valoración actual que se le otorga en la academia y en el colectivo profesional de Trabajo Social. El siguiente apartado expone la situación actual de la Sistematización y las razones que explican la expectante posición en la que se encuentra actualmente; para finalizar con un apartado en que se realizan reflexiones respecto del futuro próximo de la Sistematización en el contexto del Trabajo Social chileno. 


\section{Reflexión teórico-conceptual}

\subsection{Antecedentes históricos de la Sistematización en Chile. Desde un camino abierto a un sendero clandestino}

Tomando como primeros referentes los antecedentes históricos recopilados por Cifuentes-Gil (2010), pueden reconocerse los primeros avances respecto de la Sistematización en el año 1954, ocasión en que la Asociación Nacional de Trabajadores(as) Sociales de Estados Unidos (NASW) reflexionó en torno a la necesidad de trascender los desempeños profesionales desde actuaciones técnicas hacia la generación de un saber distintivo basado en marcos de referencia construidos desde situaciones particulares factibles de generalizar para la intervención social, aspirando a fundamentar un saber teórico propio constituido a partir de conocimientos sistematizados. Junto con estos primeros avances, en la década de 1960 surgieron nuevas propuestas teórico metodológicas desde las ciencias sociales latinoamericanas, destacando la educación popular y la investigación acción participativa como referentes identitarios (De Shutter, 1983; Freire, 1970/2000; Vio-Grossi, Gianotten y De Wit, 1988). Asimismo, se desplegaron simultáneamente avances en materias de planificación social y desarrollo comunitario, que fortalecieron los procesos formativos y ampliaron los repertorios profesionales vigentes a esa fecha (Lopera-Medina, 2014; Naciones Unidas, 1963). Todas estas propuestas fueron acogidas con gran interés por Trabajo Social, siendo sumadas al proceso de Reconceptualización que se llevaba a cabo en la época y que reflejaba un profundo cuestionamiento en torno al quehacer normativo, funcionalista y asistencial que había caracterizado tradicionalmente a la profesión (Alayón-Fernández y Molina-Molina, 2004; Jara-Holliday, 2009). En medio de estas dinámicas, la Sistematización fue identificada en su concepto y proyectada en un horizonte de desarrollo en el mediano y largo plazo, a pesar que su andamiaje inicial se encontraba en fase germinal respecto a sus fundamentos, marcos comprensivos o sus condiciones de aplicación. No obstante, recibió una positiva valoración en torno a sus potenciales aportes para Trabajo Social, instalándose rápidamente en el discurso profesional como una demanda legitimada.

Estos años de fecunda reflexión y propuesta impulsados por centros de pensamiento y organizaciones internacionales asociados a los procesos de transformación estructural económica, política y social que se llevaban a cabo en América Latina en los sectores agrarios, educacionales, sanitarios y organizacionales, fueron detenidos abruptamente a partir de la progresiva instalación de dictaduras cívico-militares en el Cono Sur durante la década de 1970, situación que ocasionó el traumático abandono de los propuestas teórico metodológicas realizadas a la fecha por las ciencias sociales de la región. Particularmente en Chile, a partir del Golpe de Estado del 11 de septiembre de 1973, se sucedieron un conjunto de acciones de censura, represión y persecución política en los claustros universitarios, especialmente en aquellos ligados a las ciencias sociales, las artes y las humanidades; junto con intervención militar de las instituciones públicas, deteniendo de esta forma la totalidad 
Castañeda-Meneses y Salamé-Coulon

de los procesos de transformación estructural en curso. Obligado por las circunstancias y en una estrategia adoptada desde una situación límite, el Trabajo Social chileno realizó un forzado viraje hacia una impronta positivista y tecnocrática, buscando contar con garantías de sobrevivencia profesional frente a un entorno académico y laboral abiertamente adverso (Castañeda-Meneses y Salamé-Coulon, 2013). Así entonces, en el Encuentro Nacional de Escuelas de Trabajo Social celebrado en noviembre de 1973 en la ciudad de Valparaíso, a escasos dos meses del Golpe Militar y en un contexto caracterizado por escuelas universitarias cerradas e intervenidas militarmente, el núcleo formativo histórico sobreviviente decide estratégicamente adscribirse al concepto de tecnología social como método único para la formación profesional. Esta propuesta se basaba en un trabajo exploratorio realizado a comienzo de los años 70 en las escuelas universitarias de Chile y que se traducía en una propuesta de un ciclo de intervención de cuatro fases secuenciales constituida por diagnóstico, programación, ejecución y evaluación, apoyada en los avances que alcanzaban la investigación cuantitativa y la planificación social en ese entonces (Aylwin, Jiménez y Quezada, 1976). En ese marco, la tecnología social era entendida como

la instrumentalización del conocimiento científico en la transformación de una realidad social que requiere modificaciones, cuyo objetivo principal es contribuir al bienestar social para satisfacer necesidades y aspiraciones de los individuos y de los grupos, promoviendo su participación organizada y consciente en acciones que les permitan superar su condición. (Figueroa, 1976, p. 148)

En consecuencia, los procesos de reflexión y cuestionamiento que venía realizando Trabajo Social debieron ser abandonados por la academia durante la larga noche de los años dictatoriales, dejando en situación pendiente los prometedores avances de nuevas propuestas teóricas metodológicas exploradas a la fecha. Esta situación también impactó a las generaciones de profesionales formadas con la impronta tecnocrática, quienes debieron replicar la racionalidad instrumental aprendida en las restringidas oportunidades de desempeño profesional provistas por las organizaciones de Estado. Dicha impronta tecnocrática despojó al Trabajo Social de sus oportunidades de reflexividad y transformación de la realidad social, aportando en cambio hacia una impecable lógica centrada en la búsqueda de la eficiencia en el proceso de intervención (Castañeda-Meneses y Salamé-Coulon, 2014a).

A pesar de ello, la Sistematización encontró un camino para mantener su vigencia durante esos años, cuando su aporte es acogido en el limitado espacio alternativo brindado por organizaciones no gubernamentales, organizaciones solidarias, centros de estudios independientes y organismos internacionales con sede en el país (Garretón, 1981). Estas instituciones alternativas, donde destacan los aportes del Centro de Investigación y Desarrollo de la Educación CIDE, y el Centro El Canelo de Nos, hicieron posible múltiples experiencias de contención, acompañamiento y denuncia en las diversas áreas críticas relacionadas principalmente con alimentación, salud, trabajo, educación, vivienda y 
Castañeda-Meneses y Salamé-Coulon

organización social en donde la dictadura cívico militar efectuaba sistemáticas y graves vulneraciones a los Derechos Humanos, Económicos y Sociales de amplios sectores de la población (Delpiano y Sánchez, 1984; García-Huidobro, Martinic y Ortiz, 1989; CastañedaMeneses y Salamé-Coulon, 2019). En dichas instancias la Sistematización fue reconocida tempranamente y acogida en forma conjunta con las experiencias de investigación acción participativa, educación popular y el despliegue inicial de la investigación cualitativa en el país, buscando rescatar y reflexionar en torno a las inéditas experiencias llevadas a cabo en la contingencia. El interés específico por la Sistematización se expresó como respuesta a la necesidad de "recuperar colectivamente la experiencia de un modo que se vincule muy concretamente a las necesidades de la práctica" (Chateau, 1982, p. 52). Esta tarea se consideraba prioritaria, dado que sus resultados se proyectaban como aportes centrales para los fundamentos de las políticas sociales que el país requeriría para un futuro escenario de transición democrática.

Para apoyar estas aspiraciones, las organizaciones alternativas realizaban proyectos de investigación, publicaciones en documentos de trabajo de circulación restringida y encuentros de intercambio y reflexión a nivel local y nacional, concluyendo que las oportunidades que representaba la Sistematización eran incuestionables en su valía, fácilmente reconocidas desde el terreno e integradas rápidamente al discurso en su carácter potencial de producir conocimientos desde la acción social o la práctica educativa que los fundaba. Pero los eventuales avances que podían realizarse sobre esta base se veían truncados frente al problema metodológico que representaba la Sistematización, ya que carecía de esquemas de trabajo que fueran ampliamente reconocidos y validados para hacer posible el proceso de transformar la acción directa a conocimiento comunicable (Palma, 1992; Valdés-Echeñique, 1992; Zúñiga, 1992). Asimismo, se apreciaban dificultades respecto al lugar que debía ocupar la dimensión teórica y conceptual en el proceso de Sistematización y en las tensiones producidas entre el relato de la experiencia directa y la construcción de categorías de mayor abstracción que mediaran en sus posibilidades de análisis y comunicación (Gajardo, 1982). No obstante, los avances en esta etapa permiten la propuesta de un concepto de Sistematización que recoge los acuerdos alcanzados a esa fecha.

\begin{abstract}
La Sistematización es un proceso metodológico, cuyo objeto es que el educador o promotor de un proyecto recupere su relación con la acción, organizando lo que sabe de su práctica para darla a conocer a otros. Este proceso supone que el sujeto piensa y actúa al mismo tiempo y que uno de los resultados de su práctica es incrementar lo que sabe de la misma. (Martinic y Walker, 1988, p. 8)
\end{abstract}

En ese entonces, los avances respecto de la Sistematización en el contexto latinoamericano eran liderados por organizaciones profesionales tales como el Centro Latinoamericano de Trabajo Social CELATS, Centro de Educación de Adultos para América Latina CEAAL y el Centro de Estudios y Publicaciones ALFORJA. Sin embargo, dadas las condiciones de la época sus aportes eran de acceso y divulgación limitada. A nivel nacional, 
la Sistematización se enlazaba como analogía abierta con otros conceptos y modalidades que buscaban definir la acción social alternativa desarrollada en resistencia, tales como proyectos de acción social, proyectos educativos, educación en grupos populares, investigación acción participativa, acción educativa, reflexión acción y similares, lo que seguía aportando confusiones y cimentando la curiosa paradoja de ser reconocida claramente como una demanda profesional legitimada que se desdibujaba en su aplicabilidad por carecer de un soporte metodológico distintivo que permitiera su concreción (Aguayo-Cuevas, 1992; Martinic y Walker, 1988; Quiroz y Morgan, 1988).

A contar de marzo de 1990, el progresivo retorno a la democracia en Chile comenzó a superar incipientemente las condiciones de censura que afectaban a las ciencias sociales en la universidad. En forma conjunta se manifestó una potente demanda por profesionales del área social para la implementación de las renovadas políticas públicas, orientadas prioritariamente a la superación de las condiciones de pobreza que afectaban a la población. Si bien, en una primera aproximación las posibilidades de la academia de acoger los avances en torno a la Sistematización realizados en las décadas anteriores era una opción posible, esto no sucedió, debido a que se manifestaron nuevas exigencias en el funcionamiento universitario ligadas directamente a lógicas neoliberales que priorizaron la realización de proyectos de investigación social competitivos orientados a la adjudicación de fondos concursables de carácter nacional e internacional, y que presionan conjuntamente por un correlato de publicaciones científicas en revistas indexadas. Ambos indicadores de productividad científica se han situado en la base de la calidad universitaria, concentrando el interés académico en la investigación social, y en consecuencia, desplazando o desvalorizando cualquier otro tipo de propuestas que no respondan a estas exigencias, situación que se mantiene sin variaciones a la fecha (Consejo Nacional de Educación de Chile [CNED], 2004). Como contraparte, el desempeño de Trabajo Social fue profundizado en las lógicas tecnocráticas de las políticas públicas, generando nuevos saberes ligados a la gestión social, por lo que la formación profesional no enfrentó cambios estructurales en sus definiciones de contenidos en las décadas posteriores, resguardando así la pertinencia de la empleabilidad de las nuevas generaciones. En ese marco, se relegaron nuevamente las oportunidades de la Sistematización, no pudiendo alcanzar el reconocimiento de su aporte distintivo para ser considerada como contenido relevante en las formaciones de nuevos cuadros profesionales. Paradojalmente, debe mencionarse que en el marco de políticas públicas tecnocráticas, han existido ocasiones en que se han ofrecido licitaciones desde organismos del Estado ligados a la ejecución directa de programas y proyectos sociales que convocan a la realización de sistematizaciones de las experiencias realizadas. En estas propuestas se han definido referenciales metodológicos amplios para la ejecución de la tarea, principalmente desde las lógicas de transcripción, registro y narración, sin presentar mayores avances respecto de concepciones que convoquen profundizar procesos en torno al análisis y la reflexión (Fondo de Solidaridad e Inversión Social [FOSIS], 2016). 
Castañeda-Meneses y Salamé-Coulon

En este período pueden identificarse dos situaciones de excepción que convocaron interés sobre la Sistematización. La primera sucedió en el año 1992, cuando la Revista de Trabajo Social de la Pontificia Universidad Católica de Chile, que a esa fecha constituía la principal publicación especializada del país, dedica un número especial a la Sistematización, presentando un conjunto de artículos cuyas autorías académicas aportan una panorámica respecto a sus fundamentos teóricos, conceptuales y éticos; junto con reflexiones sobre sus rezagos, sus desafíos futuros y sus proyecciones. En este recopilatorio, las potenciales alternativas metodológicas en torno a la Sistematización no son revisadas. Al año siguiente, el Centro de Investigación y Desarrollo de la Educación CIDE, publicó una propuesta metodológica denominada Manual para la Sistematización de proyectos educativos de acción social (Santibañez y Cárcamo, 1993) que constituyó un importante avance en esta materia. Su principal público objetivo fue definido para equipos que realizaban su acción educativa en organizaciones sociales de base y su formato se caracterizó por constituir un set de instrumentos prácticos operacionalizados a partir de pautas y guías de ejercicios. Si bien el aporte resultó relevante en el contexto metodológico, siendo reconocido en el colectivo profesional de intervención social que tuvo la oportunidad de conocer la propuesta, su impacto a nivel académico fue prácticamente imperceptible. A lo largo de la década de 1990 e inicios de la década del 2000 la lógica tecnocrática continuó liderando a Trabajo Social en la formación y en el desempeño profesional (Castañeda-Meneses y Salamé-Coulon, 2014b). En consecuencia, la Sistematización siguió ausente de la academia, a excepción de escasas e incipientes experiencias exploratorias realizadas en algunas escuelas universitarias tradicionales (Castañeda-Meneses, 2015). Sin embargo, siguió presente incansablemente en los discursos profesionales que la reconocían; comenzando a ser solicitada por algunas instituciones, asociadas a formatos de informes técnicos desde pautas de trabajo abiertas y marcadamente descriptivas.

\subsection{Situación actual de la Sistematización en Chile. Avanzar desde los senderos del miedo y la indiferencia hasta retomar la propia travesía}

La llegada de un nuevo milenio encontró a la Sistematización en Chile circulando difusamente en los discursos profesionales y sin soportes metodológicos nítidos. Su concepto se había desdibujado progresivamente y comenzaba a ser reconocido como sinónimo amplio para cualquier esfuerzo vinculado a sistemas de registros, ordenamiento, organización o transcripción de información. Con ello, su potencial había retrocedido, desplazado por otras estrategias de producción de conocimiento que contaban con mayores fundamentos teóricos conceptuales y cuyas convenciones metodológicas estabilizadas resultaban fácilmente reconocibles en el contexto de las ciencias sociales. No obstante dichas dificultades, siguió sosteniendo intacta su promesa de aportar a Trabajo Social las oportunidades de visibilizar y poner en valor su tarea profesional. Esta situación se mantuvo sin variaciones hasta mediados de la década del 2000, ocasión en la que se 
Castañeda-Meneses y Salamé-Coulon

sucedieron una serie de eventos político estratégicos en el ámbito académico que reposicionaron a la Sistematización con un mayor protagonismo.

Como antecedentes, debe precisarse que a contar del año 1990 el Ministerio de Educación chileno ha impulsado permanentemente inversiones en diversos formatos de proyectos institucionales y convenios de desempeño para todo el sistema, buscando aportar a la calidad de la educación y mejorar los déficits del sistema. Específicamente, en la educación superior la inversión se ha focalizado en infraestructura, tecnología, formación avanzada de cuadros académicos, movilidad académica y estudiantil, rediseño curricular y trabajo en red; apuntando a mejorar indicadores de gestión académica e institucional asociados a los procesos de acreditación de la calidad de las diversas instituciones y sus respectivas escuelas universitarias. En ese marco, el año 2004 se presenta una postulación conjunta de nueve escuelas de Trabajo Social, la que es adjudicada con el Proyecto en Red MECESUP UCM0401 para el período 2004-2006, con un seguimiento de cinco años. Las escuelas participantes pertenecían a las siguientes instituciones: Universidad de Antofagasta, Universidad de Valparaíso, Universidad Tecnológica Metropolitana, Universidad Católica del Maule, Universidad de Concepción, Universidad del Bío Bío, Universidad Católica de Temuco, Universidad de La Frontera y Universidad de Los Lagos. Todas ellas constituyeron una Red de Escuelas de Trabajo Social pertenecientes a las Universidades tradicionales dependientes del Consejo de Rectores de Chile CRUCH (MECESUP, 2004). El trabajo colaborativo de estas escuelas generó un perfil de egreso único por competencias, definido como un cuadro de diálogo común que facilitara la movilidad estudiantil entre instituciones. La formulación del perfil consideró básicamente informes de investigación, opinión de estudiantes, titulados(as), instancias empleadoras, equipos docentes y publicaciones en revistas disciplinarias nacionales e internacionales. Sobre la base del perfil común, cada escuela agregó sus singularidades asociadas a su historia, su territorio, sus opciones epistemológicas u otros aspectos identitarios. Dentro del perfil común de nueve competencias, fue declarada como competencia de egreso una formulación explícita referida a la Sistematización, definida en los siguientes términos "Generar conocimientos a través de las estrategias de investigación y Sistematización, contribuyendo con innovación al desarrollo disciplinario" (Castañeda-Meneses y Salamé-Coulon, 2010, p. 65). Incluir Sistematización en esta competencia se respaldaba en los avances puntuales que a este respecto se llevaban a cabo en algunas de las escuelas que formaban parte de la red, en el marco de prácticas profesionales y proyectos de titulación de pregrado. La propuesta tuvo rápida acogida debido a que, por una parte, el discurso de la Sistematización como oportunidad de construcción de conocimientos para Trabajo Social se encontraba fuertemente enraizado; y por otra, su inclusión prometía mayor dinamicidad a las prácticas profesionales en contextos de rediseño curricular. Dado que la propuesta fue considerada una plataforma de trabajo común, se respetaron las decisiones autónomas de cada escuela para ajustar la propuesta original con ciertos márgenes de convergencia. No obstante, se solicitaba encarecidamente resguardar las equivalencias propias del esfuerzo de la 
Castañeda-Meneses y Salamé-Coulon

construcción colectivas de un perfil, de tal forma de asegurar el logro de los objetivos del proyecto en red. Estas consideraciones ofrecieron una plataforma de estabilidad para incluir la Sistematización formalmente en las nuevas propuestas curriculares de las escuelas participantes (Castañeda-Meneses y Salamé-Coulon, 2010).

En consecuencia, las nueve escuelas asociadas en red iniciaron sus procesos particulares de rediseño curricular, acogiendo total o parcialmente la propuesta de incluir a la Sistematización dentro de sus definiciones de perfiles de egreso y mallas curriculares, visibilizándola expresamente a través de asignaturas y contenidos específicos; junto con reconocer formalmente los avances que a este respecto se habían desarrollado a la fecha en el país y a nivel latinoamericano, impulsando con ello un renovado interés teórico conceptual y metodológico al respecto. Dado que esta experiencia de innovación curricular en red fue valorada en forma positiva en el colectivo académico nacional, rápidamente se transformó en un referente de consulta y análisis para otras escuelas de Trabajo Social chilenas, que no habiendo participado directamente de la experiencia, consideraron sus resultados para sus propios rediseños curriculares. Con ello, la impronta de la Sistematización se expandió en todo el sistema, comenzando a ser reconocida como una alternativa posible, aun arrastrando las dificultades heredadas de su trayectoria histórica. Este reconocimiento se instaló con mayor fuerza en las escuelas profesionales ubicadas en las diversas provincias de Chile, y con menor visibilidad en las unidades académicas ubicadas en la ciudad capital.

En Marzo del 2020 se realizó una revisión a la oferta formativa de las páginas web de cada escuela de Trabajo Social de Chile, comprobando que de un total de 33 unidades académicas que conforman el sistema universitario público/privado que ofrece la carrera en el país ${ }^{1}$, 20 de ellas declaran explícitamente la Sistematización en su programa formativo, en modalidades de asignatura, unidades dentro de programas de asignatura y requerimientos de informes de Sistematización en prácticas profesionales. A partir de estos datos puede inferirse que la Sistematización ha avanzado en aproximadamente quince años desde una completa omisión en las decisiones formativas de Trabajo Social, hacia la consideración explícita en la mayoría de los programas de estudios vigentes. Las reflexiones académicas actuales la sitúan alternativamente dentro del paradigma comprensivo, homologándola con variantes y enfoques de investigación cualitativa; o como parte del paradigma crítico, en su condición de propuesta latinoamericana distintiva capaz de ofrecer relaciones dinámicas entre teoría y práctica; reflexión y acción; conocimiento y transformación. Las redes conceptuales plantean la intervención social, la práctica social y la acción social como los referentes complementarios asociados al proceso.

\footnotetext{
1 Se declaran 33 escuelas dependientes de Universidades Públicas y Privadas de Chile que cuentan con la carrera de Trabajo Social conducente al título profesional de Trabajador(a) Social y la Licenciatura en Trabajo Social como oferta institucional a marzo del 2020. El dato no está desagregado por programas y sedes.
} 
Castañeda-Meneses y Salamé-Coulon

Asimismo, debe señalarse que a partir del año 2013 los procesos de acreditación de calidad de los programas de Magister en el país aportaron criterios de evaluación diferenciados para los programas de postgrado que posean carácter académico, por estar orientados hacia el conocimiento avanzado en el área de estudio; y programas de postgrado con carácter profesional focalizados en la profundización, especialización o aplicación práctica del área de estudios definida (Comisión Nacional de Acreditación de Chile [CNA], 2013). Para la primera opción se ha mantenido para la finalización del programa, el desarrollo de un proyecto de investigación en modalidad Tesis. Para la segunda opción se ha ampliado el repertorio metodológico de las actividades posibles de realizar para finalizar el programa, bajo la denominación de Actividad Formativa Equivalente (AFE), incluyendo junto con la investigación social, las alternativas de diagnóstico social, proyecto social y Sistematización, como las de más recurrente referencia. En el caso específico de los Magister profesionalizantes asociados a escuelas de Trabajo Social de Chile, todos los programas pueden considerar a la Sistematización como actividad formativa equivalente que respalda la respectiva postgraduación. En este ámbito del post grado la Sistematización ha recibido un creciente interés en su aprendizaje y desarrollo, incluyendo a las diferentes disciplinas de las ciencias sociales de manera transversal.

Asimismo, en la última década la Sistematización ha presentado interesantes avances teórico metodológicos a nivel nacional, con publicaciones que aportan en los procesos formativos de pre y postgrado (Castañeda-Meneses, 2014; Cifuentes-Gil y PantojaKauffmann, 2019; Suarez-Manrique, 2016), complementando las contribuciones realizadas a nivel latinoamericano, a las que se puede acceder actualmente en forma más expedita a través de bases de datos especializadas. De la misma forma, debe señalarse que las experiencias de Sistematización han sido recepcionadas favorablemente en formatos de artículos científicos aceptados en revistas especializadas de Trabajo Social tanto en Chile como en Latinoamérica. Todos estos avances han contribuido en la redefinición de la posición de la Sistematización en un espectro que ofrece mayores proyecciones en su desarrollo. No obstante, se mantiene la necesidad permanente de reforzar su acervo teórico, conceptual y metodológico a partir de las experiencias realizadas a la fecha, preocupación analizada en el marco de la primera reunión académica celebrada en noviembre del año 2020 y que convocó a las escuelas de Trabajo Social de Chile que consideran a la Sistematización dentro de su oferta formativa.

\section{Conclusiones. Ya he visto este camino antes. Me conduce a tu puerta}

La referencia histórica ha permitido presentar los principales hitos que han dificultado la incorporación de la Sistematización a la formación profesional de Trabajo Social en Chile. En ese marco, pueden declararse algunos factores complementarios que también influyen en este sentido. Un primer factor está asociado al juicio desfavorable que se realiza en torno a la Sistematización en sus oportunidades de aportar a la construcción de conocimiento 
Castañeda-Meneses y Salamé-Coulon

disciplinario, cuando es analizada a la luz de los avances teórico metodológicos del repertorio disponible en ciencias sociales. Engañosamente, pudiera considerarse una alternativa de menor rango frente al avance alcanzado por la investigación social o la investigación evaluativa. Lo que se ignora en ese juicio, son los tiempos transcurridos para la consolidación y supremacía investigativa, que a la fecha superan ampliamente un siglo de desarrollo, tiempo que ha avalado sus avances, cuestionamientos y desaciertos, para mostrar en la actualidad convenciones plenamente validadas y aceptadas por la comunidad científica. Los tiempos involucrados en el desarrollo histórico de la Sistematización son claramente más breves y han estado atravesados por contextos sociopolíticos adversos que han detenido sus avances y retrasado su consolidación. Por ello, la aceptación en la academia es un dato clave, ya que le otorga la posibilidad de ser respaldada por la formación universitaria en nuevas generaciones de profesionales que posean una impronta clara respecto a su valor y que puedan contribuir a su consolidación futura. Asimismo, el espacio académico constituye una zona protegida de exploración, creación, validación y reflexión teórico metodológica, capaz de aportar crecientes estabilidades para su despliegue. Por lo tanto, los acontecimientos de las dos últimas décadas develan la paradoja del rechazo de la academia por la Sistematización por su rezago frente a la investigación social; que trae como consecuencia no contar con avances reconocidos por la academia para poder ser incorporada como referente formativo en el corto plazo. Dicho de otra forma, como en un círculo sin fin, la causa de su rechazo es la causa de su rezago.

Un segundo factor se relaciona con la clásica valoración diferenciada entre teoría y práctica, en donde la academia se asocia directamente con la primera y el colectivo profesional en ejercicio se vincula a la segunda. En ese orden de valoración, la academia no prioriza la práctica como foco de interés y por lo tanto, tampoco prioriza el desarrollo teórico metodológico de una propuesta que se relaciona con ella en forma directa. Dado que las actividades universitarias alejan progresivamente a los equipos académicos de las contingencias propias de la práctica, sus competencias para describirla, analizarla y reflexionar sobre ella disminuyen en su destreza e interés. Por oposición, el colectivo profesional se aleja de la academia en su trayectoria laboral y prioriza a la práctica como foco de interés. Sus competencias para describirla, analizarla y reflexionar sobre ella aumentan, necesitando de una propuesta teórico metodológica ajustada a su experiencia práctica que le permita desplegar sus aprendizajes. Si un equipo académico no reconoce con pertinencia el valor de la práctica, no puede comprender plenamente la importancia de fortalecer la Sistematización como parte del repertorio del colectivo profesional.

Un tercer factor a considerar es el desafío de formar en Sistematización a estudiantes de pregrado que si bien pueden recibir formalmente los contenidos, carecen de la experiencia profesional suficiente para la debida comprensión de las implicancias del proceso de Sistematización. No obstante, la decisión de considerar sus contenidos en el pregrado representa una opción de modelamiento del proceso y de instalación de 
Castañeda-Meneses y Salamé-Coulon

habilidades iniciales que progresivamente se fortalecerán en el contacto permanente con la experiencia profesional.

En términos de sus desafíos futuros, la Sistematización requiere continuar profundizando sus fundamentos teóricos conceptuales y validando sus propuestas metodológicas, siempre afectados por la esquiva legitimación otorgada por la academia y por la incapacidad de ofrecer en forma plena los esquemas operativos requeridos por el colectivo profesional de Trabajo Social, que la sigue reconociendo y aguardando pacientemente.

A modo de corolario, puede plantearse que desde su génesis la Sistematización en Chile ha transitado por un largo y sinuoso camino, adverso en algunos tramos, clandestino en otros recodos, prometedor en los tramos en los que ha podido y la han dejado avanzar. Ha debido afrontar con serenidad el largo sendero del cuestionamiento académico realizado bajo el implacable brillo meridiano de las ciencias sociales. No obstante, no ha abandonado la marcha. Conoce el difícil camino que ya ha visto muchas veces antes, pero que siempre la sostiene y la acompaña en la perenne aspiración de construir conocimientos desde una práctica revalorizada; que conduce a la puerta de la experiencia y el aprendizaje, umbrales desde donde Trabajo Social se reconoce, se reencuentra y se fortalece a partir de su propia identidad profesional.

\section{Referencias bibliográficas}

Aguayo-Cuevas, C. P. (1992). Fundamentos teóricos de la Sistematización. Revista de Trabajo Social, (61), 31-35.

Alayón-Fernández, N., y Molina-Molina, M. (2004). Acerca del movimiento de reconceptualización. Prospectiva. Revista de Trabajo Social e intervención social, (9), 31-40.

Aylwin, A., Jiménez M., y Quezada M. (1976). Un enfoque operativo de la metodología de Trabajo Social. Santiago, Chile: Pontificia Universidad Católica de Chile.

Castañeda-Meneses, P. (2014). Propuestas Metodológicas para Trabajo Social en Intervención Social y Sistematización. Cuaderno Metodológico. Valparaíso, Chile: Universidad de Valparaíso.

Castañeda-Meneses, P. (2015). Sistematización y generación de conocimientos en Trabajo Social. Aportes metodológicos a la formación profesional. Alternativas, (22), 23-32.

Castañeda-Meneses, P., y Salamé-Coulon, A. M. (2010). Construcción colectiva del perfil académico profesional en Trabajo Social. Revista Electrónica de Desarrollo de Competencias REDEC, (5), 42-71.

Castañeda-Meneses, P., y Salamé-Coulon, A. M. (2013). Trabajo Social chileno y Gobierno Militar. 40 años de memoria y olvido. Revista de Trabajo Social, (84), 55-66. 
Castañeda-Meneses y Salamé-Coulon

Castañeda-Meneses, P., y Salamé-Coulon, A. M. (2014a). Trabajo social chileno y dictadura militar. Memoria profesional predictatorial Período 1960-1973. Agentes de cambio social y trauma profesional. Revista Rumbos TS, (9) 8-25.

Castañeda-Meneses, P., y Salamé-Coulon, A. M. (2014b). Profesionalidad del Trabajo Social chileno. Tradición y Transformación. Temuco, Chile: Ediciones Universidad de La Frontera.

Castañeda-Meneses, P., y Salamé-Coulon, A. M. (2019). Memoria profesional y Trabajo Social chileno. Derechos humanos y dictadura cívico militar. Revista Katálysis, 22(2), 284-292.

Chateau, J. (1982). Sobre la Sistematización de experiencias en la acción social. Presentación de una metodología. Recuperado de http:/ / flacsochile.org/biblioteca/pub/memoria/1982/001115.pdf.

Cifuentes-Gil, R. M. (diciembre, 2010). Sistematización de experiencias en Trabajo Social: desafío inminente e inaplazable. En II Congreso Internacional Trabajo Social Formación profesional investigación Sistematización e identidad profesional en la modernidad, preguntas y respuestas, Arequipa, Perú.

Cifuentes-Gil, R. M., y Pantoja-Kauffmann, G. F. (2019). Sistematización de experiencias para construir saberes y conocimientos desde las prácticas: Sustentos, orientaciones, desafíos. Córdoba, Argentina: Editorial Brujas.

Comisión Nacional de Acreditación de Chile. (2013) Guía para la elaboración del informe de autoevaluación de programas de magister. Recuperado de https:/ / www.cnachile.cl/SiteAssets/Lists/Acreditacion/AllItems/GUIA-PARAELABORACION-INFORME-AUTOEVALUACI\%C3\%93N-MAG\%C3\%8DSTER.pdf.

Consejo Nacional de Educación de Chile. (2004). El aseguramiento de la calidad de la educación superior. Santiago, Chile: CNED.

De Shutter, A. (1983). Investigación participativa. Una opción metodológica de la educación de adultos. México: CEFRAL.

Delpiano A., y Sánchez D. (1984). Educación Popular en Chile. 100 experiencias. Santiago, Chile: CIDE-FLACSO.

Figueroa, A. (1976). Evolución del servicio social profesional en Chile, durante el período comprendido entre los años 1925 y 1975. (Trabajo de grado). Universidad de Chile, Departamento de Ciencias Humanas y Desarrollo Social, Escuela de Servicio Social, Valparaíso, Chile.

Fondo de Solidaridad e Inversión Social. (18 de diciembre de 2020). Resultado de Licitaciones. Código de Licitación No. 1900-3-LE 16. Recuperado de https:/ / www.mercadopublico.cl/Portal/Modules/Site/Busquedas/ResultadoBusq ueda.aspx?qs=1\&IdEmpresa=SZjirnKcRts\%3d.

Freire, P. (1970/2000). Pedagogía del Oprimido. (53 ed.). México. Siglo XXI.

Gajardo, M. (1982). La Educación Popular en Chile. Un esfuerzo de Sistematización. Santiago, Chile: PIEE. 
Castañeda-Meneses y Salamé-Coulon

García-Huidobro, J., Martinic, S., y Ortiz, I. (1989). Educación popular en Chile: trayectoria, experiencias y perspectivas. Santiago, Chile: Centro de Investigación y Desarrollo de la Educación.

Garretón, M. (1981). Las ciencias sociales en Chile al inicio de los ochenta. Situación, problemas y perspectivas. Santiago, Chile: FLACSO.

Jara-Holliday, O. (2009). La Sistematización de experiencias y las corrientes innovadoras del pensamiento latinoamericano. Una aproximación histórica. Diálogo de Saberes, (3,) 118129.

Lennon J., \& McCartney, P. (1970). The long and winding road. En Let it be. [disco]. Apple Records.

Lopera-Medina, M. (2013) Aspectos históricos y epistemológicos de la planificación para el desarrollo. Revista Gerencia y Politicas de Salud, 13(26), 28-43.

Martinic S., y Walker, H. (1988) Profesionales en la acción. Santiago de Chile. CIDE

MECESUP. (2004). Proyecto MECESUP 0401 La innovación curricular como nuevo desafío de las escuelas de Trabajo Social del CRUCH. Santiago de Chile. Ministerio de Educación.

Naciones Unidas. (1963). Memorándum sobre Desarrollo Comunal. Santiago, Chile: Organización de Naciones Unidas.

Palma, D. (1992). La Sistematización como estrategia de conocimiento en la educación popular. El estado de la cuestión en América Latina. Santiago, Chile: CEAAL.

Quiroz, T., y Morgan M. (1988). La Sistematización. Un intento conceptual y una propuesta de operacionalización. Lima, Perú: CELATS, 1988.

Santibañez, E., y Cárcamo M. (1993). Manual de Sistematización de proyectos educativos de acción social. Santiago de Chile: Centro de Investigación y Desarrollo de la Educación CIDE.

Suarez-Manrique, P. (2016). La Sistematización y la producción de conocimientos en el Trabajo Social: des-atando al sujeto. Santiago, Chile: Ediciones Universidad Tecnológica Metropolitana.

Valdés-Echeñique, X. (1992). Las cuentas pendientes de la Sistematización. Revista de Trabajo Social, (61), 9-17.

Vio-Grossi, F., Gianotten, V., y De Wit, T. (1988). Investigación Participativa y Praxis Rural. Santiago, Chile: Consejo de Educación de Adultos de América Latina CEAAL.

Zúñiga, R. (1992). Sobre el sistematizar. Revista de Trabajo Social, (61), 19-29. 


\section{OTROS ARTÍCULOS DE PROSPECTIVA No. 31 DE 2021}

\section{PRESENTACIÓN}

Presentación. Reflexiones sobre desafios al publicar sistematizaciones

Rosa María Cifuentes-Gil

\section{EDITORIAL}

Reflexiones sobre Trabajo Social: aportes de la Sistematización

María Rocío Cifuentes-Patiño

\section{ARTÍCULOS}

Hacer lo que se sabe, pensar lo que se hace. La sistematización como modalidad investigativa Alfonso Torres-Carrillo

Aportes y desafios de la Sistematización de experiencias en el Trabajo Social y la extensión crítica. Apuntes y reflexiones desde la perspectiva de la Educación Popular

María Rosa Goldar

Valeria Chiavetta

La sistematización en Trabajo Social y la epistemología feminista del punto de vista. Diálogos sobre la producción de conocimiento sustentada en experiencias

Ruth Noemí Parola

María Florencia Linardelli

La Sistematización investigativa de las experiencias: del baile de los que sobran a la fiesta de los que faltan

María Belén Ortega-Senet

Sistematización y Trabajo Social en Chile. El largo y sinuoso camino

Patricia Lorena Castañeda-Meneses

Ana María Salamé-Coulon
Sentipensar la pandemia COVID-19 desde la sistematización de la experiencia en Trabajo Social: reflexiones del profesor Oscar Jara Holliday

Elia Sepúlveda-Hernández

La sistematización de experiencias, una investigación social cualitativa que potencia buenas prácticas de convivencia y gobierno. La experiencia de un conjunto residencial multifamiliar en Cali, Colombia Martha Lucia Echeverry-Velásquez Manuela Prada-Dávila

Construcción de subjetividades epistemológicaspolíticas de profesoras y profesores de Investigación social en una universidad privada y confesional en Bogotá

Giovanni Mora-Lemus

Sistematización de la experiencia Reconocimiento de los derechos humanos del adulto mayor en dos familias residentes en Cali y Valledupar (Colombia)

Lina María Cuello-Lacouture

Jimena del Pilar Jaramillo-Jaramillo

La memoria transformadora como estrategia de intervención profesional en los procesos de reconciliación social: comprensión a partir de mujeres campesinas, excombatientes y jóvenes en Manizales, Colombia

Yeimmy Stephania Corredor-Sotelo

Juliana Fuertes-Fuertes

Sistematización de una estrategia de educación informal implementada en personas privadas de la libertad en el establecimiento penitenciario de mediana seguridad y carcelario de Barranquilla, Colombia

Rafael Humberto Herrera-Mercado Rafael Alberto Zambrano-Vanegas 
Aportes significativos del proceso de intervención comunitaria con la Escuela Popular de Comunicación Alternativa Jaime Garzón de la ciudad de Cúcuta, Colombia

Carlos Lasso-Urbano

La sistematización de la intervención como metodología de investigación en Trabajo Social. Importancia práctica y teórica de la fase de recogida de datos en la intervención social según experiencia del Programa de Apoyo a las Familias en Zaragoza, España

Elisa Esteban-Carbonell

Nuria Del Olmo-Vicén

Papel de la sistematización de experiencias en los procesos de evaluación de intervenciones de salud pública en la Comuna Saludable por la Paz, Cali - Colombia

Jenny Faisury Peña-Varón

Paola Andrea Marín-Velásquez

Janeth Mosquera-Becerra

Experiencia de intervención social en hogares comunitarios integrales del barrio Alfonso Bonilla Aragón, Cali - Colombia

Julián Alexander Montaño-Cárdenas

Las políticas sociales y el gobierno de la "población indígena". Estrategias y regulaciones en el multiculturalismo chileno

Rodrigo Agustín Navarrete-Saavedra

Representaciones sociales sobre estilo de autoridad y tipos de interacción en cuidadores de residencias de protección infantil en Chile

Marcelo Gallegos-Fuentes
Carmen Gloria Jarpa-Arriagada

Reflexiones sobre inseguridad social y cuestiones penales. Una respuesta estratégica a partir de experiencias de cooperativismo con ex detenidos en Argentina

Analia Elizabeth Otero

Yael Yanina Barrera

Desarrollo y salud: la emergencia de un nuevo paradigma

Jesús María Sánchez-Ordóñez

Trabajo Social en ejercicio libre: la perspectiva profesional en España

Paula Frieiro-Padín

Tamara Fernández-Arias

Rubén González-Rodríguez

\section{RESEÑAS}

Social Work and the City: Urban Themes in 21stCentury Social Work

Felipe Saravia-Cortés

Respuestas del Trabajo Social ante emergencias sociales y problemáticas sociales complejas de México y España

Felipe Saravia-Cortés

El feminismo, el género y la profesionalización del trabajo social en Colombia (1936-2004)

Ambar Oriana Serna-Lombo

El puño invisible. Arte, revolución y un siglo de cambios culturales

Carlos Arturo Robledo-Marín

\section{PROSPECTIVA}

No. $31 \bullet$ ene.-jun. 2021

e-ISSN: 2389-993X • Universidad del Valle 